\title{
Muscle depletion in cirrhotic patients assessed using computed tomography: a cross-sectional study
}

\author{
Elisa Sfoggia Romagna', Marcelo Campos Appel-da-Silva", Eiji Suwa"', Fabio Kunihiro Maeda"v , Angelo Alves de Mattos`, \\ Cristiane Valle Tovo ${ }^{\mathrm{V}}$
}

Universidade Federal de Ciências da Saúde de Porto Alegre (UFCSPA), Porto Alegre (RS), Brazil

MD. Endocrinologist and Postgraduate Student, Department of Hepatology, Universidade Federal de Ciências da Saúde de Porto Alegre (UFCSPA), Porto Alegre (RS), Brazil.

(D) orcid.org/0000-0002-6754-1544

"MD. Gastroenterologist and Postgraduate Student, Department of Hepatology, Universidade Federal de Ciências da Saúde de Porto Alegre (UFCSPA), Porto Alegre (RS), Brazil.

(D) orcid.org/0000-0002-7139-2115

'"MD. Gastroenterologist, Hospital Nossa Senhora da Conceição (HNSC); and Radiologist, Imaging Service, Unimed Porto Alegre, Porto Alegre (RS), Brazil.

(D) orcid.org/0000-0002-5540-4200

"vphysicist, Nuclear Medicine Service, Santa Casa de Misericórdia de Porto Alegre, Porto Alegre (RS), Brazil.

(D) orcid.org/0000-0001-7654-5144

'MD. Physician, Department of Hepatology, Universidade Federal de Ciências da Saúde de Porto Alegre (UFCSPA), Porto Alegre (RS), Brazil. (D) orcid.org/0000-0003-2417-9765

"MD, PhD. Adjunct Professor and Coordinator, Postgraduate Program on Hepatology, Universidade Federal de Ciências da Saúde de Porto Alegre (UFCSPA), Porto Alegre (RS), Brazil. (D) orcid.org/0000-0002-7932-5937

KEY WORDS (MeSH terms):

Liver cirrhosis.

Sarcopenia.

Mortality.

Malnutrition.

AUTHORS' KEY WORDS:

Muscle depletion.

Cirrhosis.

Chronic liver disease

\begin{abstract}
BACKGROUND: Sarcopenia is a common complication in patients with cirrhosis and may lead to increased morbidity and mortality.

OBJECTIVE: To investigate the prevalence of sarcopenia and its association with disease severity scores, among patients with cirrhosis.

DESIGN AND SETTING: Observational and retrospective cohort study carried out in a tertiary-care hospital in southern Brazil.

METHODS: This study was conducted among patients with chronic liver disease who were followed up at the gastroenterology and hepatology outpatient clinic of a tertiary-care hospital in southern Brazil and who underwent computed tomography scans of the abdomen through any indication.

RESULTS: We included 83 patients in the study. In the population evaluated, there was a predominance of males (57.80\%) and the mean age was 56 years. Hepatitis B or C virus was present in the genesis of the disease in $34.9 \%$ of the cases, followed by an etiology of alcohol abuse (30.1\%). Sarcopenia was diagnosed in 41 (49.4\%) of the patients when the cutoff point for cirrhotic patients was used. There was no significant correlation between the Child-Pugh and MELD severity scores and the occurrence of sarcopenia.
\end{abstract}

CONCLUSION: Sarcopenia presents high prevalence among patients with chronic liver disease, without any association with predictors of severity.

\section{INTRODUCTION}

Chronic liver disease is a major global public health problem. Liver cirrhosis and hepatocellular carcinoma respectively account for over 1.2 million and 800,000 deaths annually. ${ }^{1-5}$ According to a study conducted in Brazil, liver diseases are the eighth leading cause of death among patients treated through the public healthcare system, and cirrhosis is the most prevalent type. ${ }^{6}$

Protein-energy malnutrition is frequently observed in cases of liver cirrhosis. This, in association with low physical activity, may result in sarcopenia. The prevalence of protein-energy malnutrition is around $20 \%-30 \%$ among patients with chronic liver disease and over $60 \%$ among patients with advanced cirrhosis. ${ }^{7-11}$

Sarcopenia has been described as a syndrome characterized by progressive and extensive loss of strength and skeletal muscle mass, with a risk of unfavorable outcomes, including patient morbidity and mortality. ${ }^{7,11-14}$ It is one of the most common complications in cirrhotic patients, with prevalence ranging from $30 \%$ to $70 \%$, and it involves reduced quality of life and increased infection rates. It is an independent mortality factor that implies a worse outcome after liver transplantation. ${ }^{11,15-18}$ However, the diagnostic criteria are not uniform.

The European Working Group on Sarcopenia in Older People (EWGSOP) has defined a list of diagnostic criteria. In its latest publication (2018), this group recommended that both low muscle mass and low muscle function (strength or performance) should be used to diagnose sarcopenia. ${ }^{12,19}$ The justification for using two criteria is based on the fact that muscle strength does not depend on muscle mass alone. ${ }^{12,13,19,20}$ Therefore, when only the 'muscle mass' is evaluated, the term 'muscle depletion' can be used.

On the other hand, the European Association for the Study of the Liver (EASL) recommends that, among cirrhotic patients, sarcopenia should be evaluated by means of abdominal computed tomography (CT). This parameter has been validated using dual-energy $\mathrm{x}$-ray emission densitometry, which is considered to be the gold standard. ${ }^{11}$ However, there is divergence in the 
literature regarding the best cutoff point for diagnosing sarcopenia in patients with cirrhosis, using $\mathrm{CT}^{21,22}$

\section{OBJECTIVE}

The aims of this study were to investigate the prevalence of sarcopenia among cirrhosis patients and to ascertain whether there might be an association between sarcopenia and disease severity.

\section{METHODS}

A cross-sectional study was conducted using data obtained through a review of medical records. The patients included were aged 18 years or older, had been diagnosed with cirrhosis of any etiology, had undergone abdominal CT through any indication and were being followed up at the gastroenterology and hepatology outpatient clinic of a tertiary-care hospital in Porto Alegre, southern Brazil. The vast majority of the CT scans were performed to evaluate the presence of liver lesions, but CT was also indicated for evaluating abdominal pain in patients attended at the emergency unit, and also within the routine evaluation for liver transplantation.

The exclusion criteria consisted of occurrences of cases of HIV co-infection, organ transplantation and inadequate records.

Cirrhosis was diagnosed from the clinical findings, laboratory tests, imaging examinations and/or upper digestive endoscopy, and from histopathological examinations.

The review of the medical records was based on data that had been obtained at the time when the abdominal CT scan was performed. The variables analyzed were age, sex, etiology of liver disease, Child-Pugh score, ${ }^{23}$ model of end-stage liver disease (MELD) score ${ }^{24}$ ascites at the time of CT, previous history of hepatic encephalopathy, previous history of digestive bleeding, presence of hepatocellular carcinoma (HCC) and patient outcome (death or liver transplantation).

Sarcopenia was diagnosed by means of abdominal CT scans. On these, the third lumbar vertebra (L3) was identified and the transverse area of the abdominal and paraspinal wall muscles involved (psoas, erector spinae, quadratus lumborum, transversus abdominis, internal and external oblique muscles and rectus abdominis) was measured (Figure 1). This measurement in square centimeters was divided by the patient's height squared, and is referred to as the L3 skeletal muscle index (L3 SMI). The muscle area in this region is commonly used for diagnostic purposes because it includes central skeletal muscles whose mass is independent of activity and water retention, and it corresponds best to the patient's total muscle mass. All the CT images were analyzed by the same medical physicist using the ImageJ software, which is similar in accuracy to other types of software currently used in diagnosing sarcopenia. ${ }^{25}$

Two pairs of cutoff points for L3 SMI were used for further comparisons. The first was based on Carey et al., ${ }^{26}$ who evaluated a specific cutoff point for patients with cirrhosis, and defined that sarcopenia was present when $\mathrm{L} 3 \mathrm{SMI}<50 \mathrm{~cm}^{2} / \mathrm{m}^{2}$ in men and $<39 \mathrm{~cm}^{2} / \mathrm{m}^{2}$ in women. The second was based on Prado et $\mathrm{al},{ }^{27}$ who evaluated patients with solid tumors of the respiratory and gastrointestinal tract and took cutoff points for sarcopenia of $<52.4 \mathrm{~cm}^{2} / \mathrm{m}^{2}$ for men and $<38.5 \mathrm{~cm}^{2} / \mathrm{m}^{2}$ for women. This second pair of cutoff points are the ones used by the EWGSOP. ${ }^{12,19}$

For the statistical analysis, categorical variables were described according to the frequency and percentage. The sarcopenia prevalence rate was presented with its respective $95 \%$ confidence interval (95\% CI). Normally-distributed quantitative variables were described in terms of the mean and standard deviation. Categorical variables were compared using the chi-square test or Fisher's exact test. A 5\% significance level was used for the comparisons.

The study was approved on November 8,2011, by the ethics committee of the institution involved, under the protocol number 3675/11.

\section{RESULTS}

Among the 570 cirrhotic patients seen at the outpatient clinic, 466 had not undergone abdominal CT, and there was no record of height for 21 patients. Thus, the final sample consisted of 83 patients. These were predominantly male patients $(48 ; 57.80 \%)$, with a mean age of $56.68 \pm 10.40$ years $(63.9 \%$ were younger than 60 years of age), and a mean body mass index (BMI) of $27.5 \pm 4.3 \mathrm{~kg} / \mathrm{m}^{2}$. Regarding etiology, $34.9 \%$ of the cases were due to hepatitis B or C, and $30.1 \%$ were due to alcohol abuse.

At the time of the evaluation, 40 patients (48.2\%) had ascites; 29 (35.4\%) had presented upper gastrointestinal bleeding in the past; and 28 (33.7\%) had a history of hepatic encephalopathy. The other characteristics of the population are presented in Table 1. Eight of the patients (10\%) died as a consequence of liver disease.

Sarcopenia was identified in 41 patients (49.4\%; 95\% CI 38.2\%$60.6 \%$ ) according to the cutoff point specific for cirrhotic patients; and in 40 patients (48.2\%; 95\% CI 37.1\%-59.4\%) according to the cutoff point used for oncological patients $(\mathrm{P}=0.976)$.

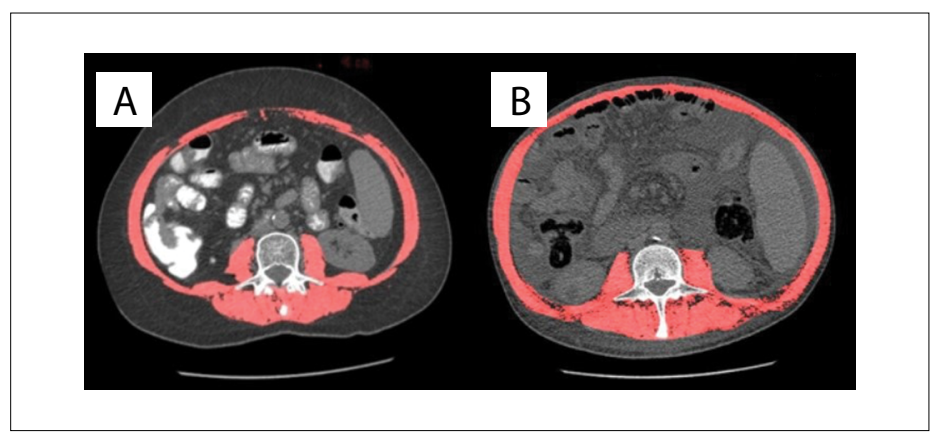

Figure 1. Cross-sectional area of third lumbar vertebra (L3) on abdominal computed tomography in a patient with sarcopenia (32.17 $\left.\mathrm{cm}^{2} / \mathrm{m}^{2}\right)(A)$ and a patient without sarcopenia $\left(52.3 \mathrm{~cm}^{2} / \mathrm{m}^{2}\right)(B)$. 
Non-obese patients $\left(\mathrm{BMI}<30 \mathrm{~kg} / \mathrm{m}^{2}\right)$ presented sarcopenia more frequently than obese patients, regardless of the cutoff point used $(\mathrm{P}=0.012$ and $\mathrm{P}=0.017$ respectively).

Among the patients with an etiology of alcohol abuse for cirrhosis, 23 (56.1\%) had sarcopenia according to the cutoff point for cirrhotic patients and 23 (57.5\%) according to the oncological cutoff point. Among patients with other etiologies, 18 cases (43.9\%) of sarcopenia were identified using the cirrhosis cutoff point and $17(42.5 \%)$ were identified using the oncological cutoff point; there was no difference between these etiology groups ( $\mathrm{P}=0.38$ and $\mathrm{P}=0.27$ respectively).

Evaluation of the patients in terms of compensated cirrhosis (Child-Pugh A) and decompensated cirrhosis (Child-Pugh B and C) showed that among the 41 patients diagnosed with sarcopenia according to the cirrhosis-specific cutoff point, 22 (53.7\%) were Child-Pugh A and 19 (46.3\%) were Child-Pugh B and C ( $\mathrm{P}=0.383)$ (Table 1). Among the 40 patients diagnosed with sarcopenia according to the oncological cutoff point, 21 (52.5\%) were Child-Pugh A and 19 (47.5\%) were Child-Pugh B and C $(\mathrm{P}=0.513)$ (Table 2).

Table 1. Clinical characteristics of the patients using the cirrhosis cutoff points ( $<50 \mathrm{~cm}^{2} / \mathrm{m}^{2}$ in men and $<39 \mathrm{~cm}^{2} / \mathrm{m}^{2}$ in women)

\begin{tabular}{|c|c|c|c|}
\hline & $\begin{array}{c}\text { With } \\
\text { sarcopenia }\end{array}$ & $\begin{array}{c}\text { Without } \\
\text { sarcopenia }\end{array}$ & $\mathbf{P}$ \\
\hline & $n=41$ & $n=42$ & \\
\hline ETIOLOGY, n (\%) & & & 0.383 \\
\hline Alcohol & $23(56.1)$ & $19(45.2)$ & \\
\hline Other & $18(43.9)$ & $23(61.1)$ & \\
\hline CHILD-PUGH, n (\%) & & & 0.383 \\
\hline A & $22(53.7)$ & $18(49.2)$ & \\
\hline$B$ and $C$ & $19(46.3)$ & $24(57.1)$ & \\
\hline \multicolumn{4}{|l|}{ MELD, n (\%) } \\
\hline$>15$ & $7(17.1)$ & $14(33.3)$ & 0.147 \\
\hline$\leq 15$ & 34 (82.9) & $28(66.7)$ & 0.147 \\
\hline
\end{tabular}

$M E L D=$ model of end-stage liver disease.

Table 2. Clinical characteristics of the patients using the oncological cutoff points ( $<52.4 \mathrm{~cm}^{2} / \mathrm{m}^{2}$ in men and $<38.5 \mathrm{~cm}^{2} / \mathrm{m}^{2}$ in women)

\begin{tabular}{lccc} 
& $\begin{array}{c}\text { With } \\
\text { sarcopenia }\end{array}$ & $\begin{array}{c}\text { Without } \\
\text { sarcopenia }\end{array}$ & $\mathbf{P}$ \\
\cline { 2 - 4 } & $\mathbf{n = 4 0}$ & $\mathbf{n = 4 3}$ & \\
$\begin{array}{l}\text { ETIOLOGY, } \mathbf{n}(\%) \\
\quad \text { Alcohol }\end{array}$ & $23(57.5)$ & $19(44.2)$ & 0.275 \\
$\quad$ Other & $17(42.5)$ & $24(55.8)$ & \\
$\begin{array}{l}\text { CHILD-PUGH, } \mathbf{n}(\%) \\
\text { A }\end{array}$ & $21(52.5)$ & $19(44.2)$ & 0.513 \\
B and C & $19(47.5)$ & $24(55.8)$ & \\
MELD, $\mathbf{n}(\%)$ & & & \\
$>15$ & $7(17.5)$ & $14(32.6)$ & 0.185 \\
$\leq 15$ & $33(82.5)$ & $29(67.4)$ & 0.185 \\
\hline
\end{tabular}

$M E L D=$ model of end-stage liver disease.
There was no significant association between MELD score and sarcopenia $(\mathrm{r}=-0.035 ; \mathrm{P}=0.147)$ using the cutoff of MELD $>15$. There was no correlation between sarcopenia and Child-Pugh score according to the cirrhosis-specific cutoff point $(\mathrm{P}=0.518)$ or the oncological cutoff point $(\mathrm{P}=0.632)$ (Table 2$)$.

There were no significant differences in the numbers of patients with upper gastrointestinal bleeding $(\mathrm{P}=1.00)$, hepatic encephalopathy $(\mathrm{P}=1.00)$ or ascites $(\mathrm{P}=1.00)$ according to the cutoff point.

Age over 60 years was not significantly associated with either cutoff point. There were 18 patients (21.7\%) with type 2 diabetes mellitus, and seven of these (17\%) had sarcopenia, which was not significant. Twenty-six patients (31.3\%) had HCC and, of these, eight (19.5\%) had sarcopenia according to the two cut-off points $(\mathrm{P}=0.040)$.

Among the eight patients who died, two had sarcopenia according to both cutoff points ( $25 \%$; $\mathrm{P}=0.275$ for the cirrhosis cutoff point; and 25\%; $\mathrm{P}=0.269$ for the oncological cutoff point).

\section{DISCUSSION}

The nutritional status of patients with cirrhosis is increasingly stressed in the literature. In recognition of the impact of muscle loss in these patients, we conducted a study to assess the prevalence of sarcopenia and its relationship with disease severity, with comparisons between different cutoff points used for making the diagnosis. We found that the prevalence of sarcopenia was $49.4 \%$ when we used a cutoff point established specifically for cirrhotic patients. Since few studies have evaluated sarcopenia in cirrhotic patients, we also used a cutoff point that had previously been evaluated for an oncological population, which resulted in a prevalence rate of $48 \%$. The results did not differ significantly between the two cutoff points.

The prevalence rate found in the present study is concordant with the findings of Jeong et al., who evaluated a similar outpatient population of 131 cirrhotic patients who underwent CT. Using a cutoff point established by Prado et al., ${ }^{27}$ they found a prevalence of $48.9 \%{ }^{28}$

Tandon et al. also evaluated cirrhotic patients (most of them compensated) in outpatient follow-up with CT or magnetic resonance imaging, and found a prevalence of $43 \%{ }^{29}$ Among non-liver transplantation cirrhotic patients evaluated using CT, Hanai et al. found a higher prevalence of sarcopenia (68\%). ${ }^{17}$

Interestingly, Montano-Loza et al. ${ }^{30}$ and Tandon et al. ${ }^{31}$ found lower prevalence of sarcopenia than what was observed in the present study ( $40 \%$ and $41 \%$, respectively). However, they evaluated patients on the liver transplantation waiting list whose condition was more severe. Likewise, Meza-Junco et al. evaluated 116 patients on liver transplantation lists and found a prevalence of sarcopenia of $30 \% .{ }^{32}$ On the other hand, Giusto et al. found a prevalence of $78 \%$ among patients who were eligible for liver transplantation. ${ }^{18}$ 
In Brazil, Zambrano et al. evaluated cirrhotic patients who were being followed up as outpatients and found a prevalence of $17 \%{ }^{33}$

There are divergences of opinion regarding which cutoff points should be used for diagnosing sarcopenia. Prado et al. evaluated patients with solid tumors of the respiratory and gastrointestinal tract and determined that cutoffs of $52.4 \mathrm{~cm}^{2} / \mathrm{m}^{2}$ for men and $38.5 \mathrm{~cm}^{2} / \mathrm{m}^{2}$ for women were associated with increased mortality. ${ }^{27}$ Jones et al. used this cut-off point among colorectal cancer patients, and Sheean et al. used it among patients with respiratory failure. ${ }^{34,35}$ This is also the cutoff point currently recommended by the EWGSOP. ${ }^{12,19}$

Among patients with liver cirrhosis, there is even greater disagreement about which cutoff point to use, and there have been changes over the years. Tandon et al., ${ }^{29,31}$ Giusto et al. ${ }^{18}$ and MontanoLoza et al. ${ }^{30}$ evaluated the prevalence of sarcopenia among cirrhotic patients using the cutoffs proposed by Prado et al. ${ }^{27}$ However, Montano-Loza et al. subsequently proposed another cutoff point for diagnosing sarcopenia in cirrhotic patients: $50 \mathrm{~cm}^{2} / \mathrm{m}^{2}$ for men and $42 \mathrm{~cm}^{2} / \mathrm{m}^{2}$ for women. ${ }^{36,37}$ A new study by the same group, this time assessing the inclusion of sarcopenia in MELD, then used cutoffs based on Martin et al. $\left(\leq 53 \mathrm{~cm}^{2} / \mathrm{m}^{2}\right.$ for men and $\leq 41 \mathrm{~cm}^{2} / \mathrm{m}^{2}$ for women with BMI $\geq 25 \mathrm{~kg} / \mathrm{m}^{2}$, and $\leq 43 \mathrm{~cm}^{2} /$ $\mathrm{m}^{2}$ for all patients with $\left.\mathrm{BMI}<25 \mathrm{~kg} / \mathrm{m}^{2}\right) .{ }^{38,39}$ The most recent study on this topic, published by Carey et al. in 2017, was a multicenter study to determine cutoffs for diagnosing sarcopenia in cirrhotic patients. A total of 396 patients were assessed at five liver transplantation centers in the United States, and the cutoff point that was most significant for detecting survival differences between the groups was $<50 \mathrm{~cm}^{2} / \mathrm{m}^{2}$ for men and $<39 \mathrm{~cm}^{2} / \mathrm{m}^{2}$ for women. ${ }^{26}$

In view of the controversy regarding the best cutoff point for diagnosing sarcopenia, we chose to evaluate the ones that are most used, i.e. the cutoffs advocated by Carey et al., obtained from cirrhotic patients, and by Prado et al., obtained from patients with solid tumors. These were compared, and no significant difference in the prevalence of sarcopenia was found between them.

Regarding the etiology of cirrhosis, $34.9 \%$ of the cases were due to hepatitis $\mathrm{B}$ or $\mathrm{C}$, which is a proportion similar to what has been described in the literature. ${ }^{30}$ However, there was no statistically significant difference regarding the prevalence of sarcopenia and etiology. Grouping patients according to whether the etiology of their cirrhosis was alcohol abuse did not result in any significant difference.

Regarding sarcopenia and predictors of mortality (Child-Pugh and MELD), some studies did not find any relationship between sarcopenia and the degree of hepatic dysfunction. ${ }^{17,31,32}$ However, others found a relationship and suggested that including sarcopenia assessment in Child-Pugh and MELD scores could improve mortality predictions among cirrhotic patients. ${ }^{31,38,40}$ In the present study, there was no correlation between sarcopenia and the
Child-Pugh and MELD severity scores, although it should be pointed out that sarcopenia was not included in the MELD score as a prognostic tool.

Although it has been shown that sarcopenia increases mortality among cirrhotic patients, ${ }^{37,41,42}$ there was no association in the present study between mortality and sarcopenia, which might be explained by the fact that the sample consisted of outpatients, with disease of lower severity.

The main factor that may have contributed towards potential study limitations was the small sample size, which perhaps contributed to the lack of significant differences between severity, mortality and the different cutoff points. Also, the varied indications for CT were potentially a source of bias concerning clinical status. In addition, the retrospective design involved inherent limitations that should be taken into account. On the other hand, the situation depicted in this study represents the real life of outpatients in a reference center.

\section{CONCLUSION}

The present study indicated that there was high prevalence of sarcopenia among individuals with cirrhosis, even if this was compensated. In addition, no difference between the cutoff points that were used to diagnose sarcopenia was found, and there was no association between the severity predictor scores (Child-Pugh and MELD) and presence of sarcopenia. Future studies with larger samples could contribute towards better understanding of this topic.

\section{REFERENCES}

1. Rowe IA. Lessons from Epidemiology: The Burden of Liver Disease. Dig Dis. 2017;35(4):304-9. PMID: 28468017; doi: 10.1159/000456580.

2. Giannousis IP, Papatheodoridis GV, Deutsch MJ, et al. The burden and recent epidemiological changes of the main chronic liver diseases in a Greek referral tertiary centre. Eur J Gastroenterol Hepatol. 2010;22(2):172-9. PMID: 19738477; doi: 10.1097/MEG.0b013e328331115b.

3. Ginés P, Quintero E, Arroyo V, et al. Compensated cirrhosis: natural history and prognostic factors. Hepatology. 1987;7(1):122-8. PMID: 3804191; doi: 10.1002/hep.1840070124

4. Zatoński WA, Sulkowska U, Mańczuk M, et al. Liver cirrhosis mortality in Europe, with special attention to Central and Eastern Europe. Eur Addict Res. 2010;16(4):193-201. PMID: 20606444; doi: 10.1159/000317248.

5. Garcia-Tsao G, Sanyal AJ, Grace ND, et al. Prevention and management of gastroesophageal varices and variceal hemorrhage in cirrhosis. Am J Gastroenterol. 2007;102(9):2086-102. PMID: 17727436; doi: 10.1111/j.1572-0241.2007.01481.x.

6. Nader LA, de Mattos AA, Bastos GA. Burden of liver disease in Brazil. Liver Int. 2014;34(6):844-9. PMID: 24422599; doi: 10.1111/liv.12470.

7. Nishikawa H, Osaki Y. Liver Cirrhosis: Evaluation, Nutritional Status, and Prognosis. Mediators Inflamm. 2015;2015:872152. PMID: 26494949; doi: $10.1155 / 2015.872152$ 
8. Fernandes SA, Bassani L, Nunes FF, et al. Nutritional assessment in patients with cirrhosis. Arq Gastroenterol. 2012;49(1):19-27. PMID: 22481682.

9. Nunes FF, Bassani L, Fernandes SA, et al. Food consumption of cirrhotic patients, comparison with the nutritional status and disease staging. Arq Gastroenterol. 2016;53(4):250-6. PMID: 27706455; doi: 10.1590/ S0004-28032016000400008.

10. Bruix J, Sherman M, Llovet JM, et al. Clinical management of hepatocellular carcinoma. Conclusions of the Barcelona-2000 EASL conference. European Association for the Study of the Liver. J Hepatol. 2001;35(3):421-30. PMID: 11592607; doi: 10.1016/s0168-8278(01)00130-1.

11. European Association for the Study of the Liver. EASL Clinical Practice Guidelines on nutrition in chronic liver disease; J Hepatol. 2019;70(1):172-93. PMID: 30144956; doi: 10.1016/j.jhep.2018.0.024.

12. Cruz-Jentoft AJ, Baeyens JP, Bauer JM, et al. Sarcopenia: European consensus on definition and diagnosis: Report of the European Working Group on Sarcopenia in Older People. Age Ageing. 2010;39(4):412-23. PMID: 20392703; doi: 10.1093/ageing/afq034.

13. Goodpaster BH, Park SW, Harris TB, et al. The loss of skeletal muscle strength, mass, and quality in older adults: the health, aging and body composition study. J Gerontol A Biol Sci Med Sci. 2006;61 (10):1059-64. PMID: 17077199; doi: 10.1093/gerona/61.10.1059.

14. Delmonico MJ, Harris TB, Lee JS, et al. Alternative definitions of sarcopenia, lower extremity performance, and functional impairment with aging in older men and women. J Am Geriatr Soc. 2007;55(5):769-74. PMID: 17493199; doi: 10.1111/j.1532-5415.2007.01140.x.

15. Sinclair M, Gow PJ, Grossmann M, Angus PW. Review article: sarcopenia in cirrhosis--aetiology, implications and potential therapeutic interventions. Aliment Pharmacol Ther. 2016;43(7):765-77. PMID: 26847265; doi: 10.1111/apt.13549.

16. Kallwitz ER. Sarcopenia and liver transplant: The relevance of too little muscle mass. World J Gastroenterol. 2015;21(39):10982-93. PMID: 26494955; doi: 10.3748/wjg.v21.i39.10982.

17. Hanai T, Shiraki M, Nishimura K, et al. Sarcopenia impairs prognosis of patients with liver cirrhosis. Nutrition. 2015;31(1):193-9. PMID: 25441595; doi: 10.1016/j.nut.2014.07.005.

18. Giusto M, Lattanzi B, Albanese C, et al. Sarcopenia in liver cirrhosis: the role of computed tomography scan for the assessment of muscle mass compared with dual-energy $X$-ray absorptiometry and anthropometry. Eur J Gastroenterol Hepatol. 2015;27(3):328-34. PMID: 25569567; doi: 10.1097/MEG.0000000000000274.

19. Cruz-Jentoft AJ, Bahat G, Bauer J, et al. Sarcopenia: revised European consensus on definition and diagnosis. Age Ageing. 2019;48(1):16-31. PMID: 30312372; doi: 10.1093/ageing/afy169.

20. Janssen I, Baumgartner RN, Ross R, Rosenberg IH, Roubenoff R. Skeletal muscle cutpoints associated with elevated physical disability risk in older men and women. Am J Epidemiol. 2004;159(4):413-21. PMID: 14769646; doi: 10.1093/aje/kwh058.

21. Shen W, Punyanitya $M$, Wang Z, et al. Total body skeletal muscle and adipose tissue volumes: estimation from a single abdominal cross-sectional image. J Appl Physiol (1985). 2004;97(6):2333-8. PMID: 15310748; doi: 10.1152/japplphysiol.00744.2004.

22. Mourtzakis M, Prado CM, Lieffers JR. A practical and precise approach to quantification of body composition in cancer patients using computed tomography images acquired during routine care. Appl Physiol Nutr Metab. 2008;33(5):997-1006. PMID: 18923576; doi: 10.1139/H08-075.

23. Pugh RN, Murray-Lyon IM, Dawson JL, Pietroni MC, Williams R. Transection of the oesophagus for bleeding oesophageal varices. $\mathrm{Br}$ J Surg. 1973;60(8):646-9. PMID: 4541913; doi: 10.1002/bjs.1800600817.

24. Malinchoc M, Kamath PS, Gordon FD, et al. A model to predict poor survival in patients undergoing transjugular intrahepatic portosystemic shunts. Hepatology. 2000;31 (4):864-71. PMID: 10733541; doi: 10.1053/ he. 2000.5852.

25. van Vugt JL, Levolger S, Gharbharan A, et al. A comparative study of software programmes for cross-sectional skeletal muscle and adipose tissue measurements on abdominal computed tomography scans of rectal cancer patients. J Cachexia Sarcopenia Muscle. 2017;8(2):285-97. PMID: 27897414; doi: 10.1002/jcsm.12158.

26. Carey EJ, Lai JC, Wang CW, et al. A multicenter study to define sarcopenia in patients with end-stage liver disease. Liver Transpl. 2017;23(5):625-33. PMID: 28240805; doi: 10.1002/lt.24750.

27. Prado $C M$, Lieffers JR, McCargar $\sqcup$, et al. Prevalence and clinical implications of sarcopenic obesity in patients with solid tumours of the respiratory and gastrointestinal tracts: a population-based study. Lancet Oncol. 2008;9(7):629-35. PMID: 18539529; doi: 10.1016/S1470-2045(08)70153-0.

28. Jeong JY, Lim S, Sohn JH, et al. Presence of Sarcopenia and Its Rate of Change Are Independently Associated with Long-term Mortality in Patients with Liver Cirrhosis. J Korean Med Sci. 2018;33(50):e299. PMID: 30534029; doi: 10.3346/jkms.2018.33.e299.

29. Tandon P, Low G, Mourtzakis M, et al. A Model to Identify Sarcopenia in Patients With Cirrhosis. Clin Gastroenterol Hepatol. 2016;14(10):14731480.e3. PMID: 27189915; doi: 10.1016/j.cgh.2016.04.040.

30. Montano-Loza AJ, Meza-Junco J, Prado CM, et al. Muscle wasting is associated with mortality in patients with cirrhosis. Clin Gastroenterol Hepatol. 2012;10(2):166-73. PMID: 21893129; doi: 10.1016/j. cgh.2011.08.028.

31. Tandon P, Ney M, Irwin I, et al. Severe muscle depletion in patients on the liver transplant wait list: its prevalence and independent prognostic value. Liver Transpl. 2012;18(10):1209-16. PMID: 22740290; doi: 10.1002/It.23495.

32. Meza-Junco J, Montano-Loza AJ, Baracos VE, et al. Sarcopenia as a prognostic index of nutritional status in concurrent cirrhosis and hepatocellular carcinoma. J Clin Gastroenterol. 2013;47(10):861-70. PMID: 23751844; doi: 10.1097/MCG.0b013e318293a825.

33. Zambrano DN, Xiao J, Prado CM, Gonzalez MC. Patient-Generated Subjective Global Assessment and Computed Tomography in the assessment of malnutrition and sarcopenia in patients with cirrhosis: Is there any association? Clin Nutr. 2019; pii: S0261-5614(19)30274-2. PMID: 31307841; doi: 10.1016/j.clnu.2019.06.018. 
34. Jones KI, Doleman B, Scott S, Lund JN, Williams JP. Simple psoas crosssectional area measurement is a quick and easy method to assess sarcopenia and predicts major surgical complications. Colorectal Dis. 2015;17(1):O20-6. PMID: 25328119; doi: 10.1111/codi.12805.

35. Sheean PM, Peterson SJ, Gomez Perez S, et al. The prevalence of sarcopenia in patients with respiratory failure classified as normally nourished using computed tomography and subjective global assessment. JPEN J Parenter Enter Nutr. 2014;38(7):873-9. PMID: 23980135; doi: 10.1177/0148607113500308.

36. Montano-Loza AJ. Clinical relevance of sarcopenia in patients with cirrhosis. World J Gastroenterol. 2014;20(25):8061-71. PMID: 25009378; doi: 10.3748/wjg.v20.i25.8061.

37. Montano-Loza AJ, Meza-Junco J, Prado CMM, et al. New cutoff values for sarcopenia for predicting 6-month mortality in cirrhotic patients. J Hepatol. 2013;58:S63-227. doi: 10.1016/S0168-8278(13)60223-8.

38. Montano-Loza AJ, Duarte-Rojo A, Meza-Junco J, et al. Inclusion of Sarcopenia Within MELD (MELD-Sarcopenia) and the Prediction of Mortality in Patients With Cirrhosis. Clin Transl Gastroenterol. 2015;6(7):e102. PMID: 26181291; doi: 10.1038/ctg.2015.31.

39. Martin L, Birdsell L, Macdonald N, et al. Cancer cachexia in the age of obesity: skeletal muscle depletion is a powerful prognostic factor, independent of body mass index. J Clin Oncol. 2013;31(12):1539-47. PMID: 23530101; doi: 10.1200/JCO.2012.45.2722.

40. Kim HY, Jang JW. Sarcopenia in the prognosis of cirrhosis: Going beyond the MELD score. World J Gastroenterol. 2015;21(25):7637-47. PMID: 23167066; doi: 10.3748/wjg.v21.i25.7637.

41. Nishikawa H, Kita R, Kimura T, et al. Clinical implication of performance status in patients with hepatocellular carcinoma complicating with cirrhosis. J Cancer. 2015;6(4):394-402. PMID: 25767611; doi: 10.7150/ jca. 11212.

42. Fujiwara N, Nakagawa H, Kudo Y, et al. Sarcopenia, intramuscular fat deposition, and visceral adiposity independently predict the outcomes of hepatocellular carcinoma. J Hepatol. 2015;63(1):131-40. PMID: 25724366; doi: 10.1016/j.help.2015.02.031.

Authors' contributions: All authors contributed equally to this paper with conception, analysis of the data, drafting, revision and approval of the final version. All authors agree to be accountable for all aspects of the work, so as to ensure that questions relating to the accuracy or integrity of any part of the work are appropriately investigated and resolved

\section{Sources of funding: None}

Conflicts of interest: None
Date of first submission: October 7, 2019

Last received: December 3, 2019

Accepted: December 19, 2019

\section{Address for correspondence:}

Cristiane Valle Tovo

Rua Sarmento Leite, 245

Porto Alegre (RS) - Brasil

CEP 900050-170

Tel. (+55 51) 3214-8158

E-mail: cris.tovo@terra.com.br 